\title{
Desempenho em FI com Humanos: Efeito da Interação da Resposta de Consumação e do Tipo de Instrução
}

\author{
Carlos Eduardo Costa \\ Carlos Henrique Patsko \\ Rodrigo Morande Becker \\ Universidade Estadual de Londrina
}

\begin{abstract}
RESUMO
O estudo investigou o efeito da resposta de consumação sobre o desempenho em FI. No Experimento I, seis universitários foram expostos a um FI 30 s por três sessões de 20 minutos cada. Para metade dos participantes (Grupo SR-I) não foi exigida uma resposta de consumação para que pontos fossem creditados ao contador e para a outra metade uma resposta de consumação foi exigida e uma instrução "adicional" sobre esta resposta foi fornecida (Grupo CR-I). Os resultados não indicaram um efeito claro da resposta de consumação. No Experimento II oito universitários foram expostos a um FI $30 \mathrm{~s}$ por três sessões de 30 minutos cada. Ambos os grupos receberam a mesma instrução mínima sobre a tarefa experimental. O desempenho da maioria dos participantes do Grupo CR-II pareceu ficar sob controle do FI e a maioria dos do Grupo SR-II tiveram um desempenho em taxas altas. Os resultados sugerem que a interação entre a exigência de uma resposta de consumação e o tipo de instrução pode favorecer o responder em taxa baixa sob FI.
\end{abstract}

Palavras-chave: esquemas de reforçamento; intervalo fixo; resposta de consumação; instruções; humanos.

\section{ABSTRACT \\ Performance in FI with Humans: Effect of the Interaction of the Consummatory Response and the Type of Instructions}

The study investigated the effect of the consummatory response on the human performance in FI. In Experiment I, six university students were exposed to a 30-second FI schedule, during three 20minute sessions. Half the participants (SR-I Group) were not required to emit a consummatory response in order to credit the points on the earnings counter; the other half were required to emit a consummatory response, and "additional" information about it were provided (CR-I Group). The results did not point to a clear effect of the consummatory response. In Experiment II, eight university students were exposed to a 30-second FI schedule, during three 30-minute sessions. Both groups received the same minimal instructions about the experimental task. The performance of most of the participants in the CR-II Group seemed to be under the control of FI, and most of the SR-II Group had high rates of responding. The results suggest that an interaction between the requirement of a consummatory response and the kind of instruction can favor low rates of responding under FI.

Keywords: schedules of reinforcement; fixed interval; consummatory response; instructions; humans.

Em um programa de reforço em FI a primeira resposta emitida após um período designado de tempo é seguida por um evento reforçador (Catania, 1998; Ferster \& Skinner, 1957; Lattal, 1991). Dois padrões de responder sob uma contingência de FI têm sido mais comumente relatados na literatura experimental com sujeitos não-humanos. Um padrão consiste de uma pausa, logo após o reforço, seguida por um res- ponder positivamente acelerado dentro do intervalo até a liberação do próximo reforço. Este padrão tem sido denominado de scallop (Ferster \& Skinner, 1957). Outro padrão, denominado break-and-run, consiste de uma pausa, geralmente após o reforço, e uma transição abrupta na taxa de respostas de uma freqüência próxima de zero para uma freqüência constante que é mantida até o final do intervalo 
(Cumming \& Schoenfeld, 1958) ${ }^{1}$. Alguns estudos experimentais com humanos (e.g., Buskist, Miller e Bennett, 1980; Costa, Banaco \& Becker, 2005; Okouchi, 2002; Weiner, 1965, 1969) têm demonstrado variabilidade no padrão de responder entre participantes em FI. Os padrões encontrados podem variar, de taxa de respostas alta e constante; de taxa baixa - muitas vezes de uma a três respostas no final do intervalo -; de taxas "intermediárias" que podem ser relativamente constantes (i.e., sem pausa pós-reforço) ou com alguns padrões de scallop ou break-and-run.

Há uma vasta literatura na área que procura apontar e discutir as diferenças de humanos e não-humanos respondendo sob programas de reforço em geral - e sob FI, em particular (e.g., Lowe, 1979; Perone, Galizio \& Baron, 1988; Wanchisen, 1990; Weiner, 1983). Aparentemente, as hipóteses levantadas pelos diversos autores para explicar estas diferenças podem ser agrupadas em três grandes conjuntos (não mutuamente excludentes): (1) o papel do comportamento verbal (o efeito de regras e auto-regras sobre o desempenho de humanos em programas de reforço - ver, por exemplo, Bentall, Lowe, \& Beasty, 1985; Buskist, Bennett, \& Miller, 1981; Catania, 1998; Lowe, 1979); (2) as diferenças na história comportamental entre humanos e não-humanos e mesmo entre diferentes participantes humanos (e.g., Costa, 2004; Wanchisen, 1990; Wanchisen, Tatham, \& Mooney, 1989; Weiner, 1964; 1965; 1969; 1983) e (3) as variações nos procedimentos experimentais empregados nas pesquisas com humanos (tanto em relação àquelas conduzidas com nãohumanos quanto em relação às diferentes pesquisas com humanos - ver, por exemplo, Costa e cols., 2005; Matthews, Shimoff, Catania \& Sagvolden, 1977; Perone e cols., 1988; Stoddard, Sidman, \& Brady, 1988).

Stoddard e cols. (1988) conduziram um estudo no qual participaram 17 profissionais de um hospital psiquiátrico. A conseqüência programada era fichas trocáveis por mercadorias em uma loja do hospital. Em média, as fichas renderam aos participantes cerca de U\$ 3,00 por hora nas sessões experimentais. O procedimento foi dividido em quatro fases. Na primeira fase o programa de reforço era um múltiplo FR 10FI 30 s. Na segunda fase o múltiplo foi alterado para FR 50-FI $60 \mathrm{~s}$. Na terceira fase o valor de cada ficha foi dobrado e ao múltiplo FR 50-FI $60 \mathrm{~s}$ foi acrescentado um limited hold ( $\mathrm{LH}$ ) de $5 \mathrm{~s}$ que diminuiu, ao longo de 10 semanas, até $0,5 \mathrm{~s}$. Na fase final o LH foi retirado. Nenhuma restrição foi feita em relação à troca de informações entre os participantes da pesquisa ou ao uso de relógio durante a sessão experimental.
Os resultados sugeriram que o comportamento dos participantes foi sensível à contingência de reforço uma vez que (a) o responder durante o componente de FI mudou de um padrão de responder constante para um padrão de break-and-run ou de uma resposta por intervalo com a exposição continuada às contingências de reforço; (b) as taxas e padrões do responder diferiram em cada componente do programa de reforço múltiplo; (c) as pausas durante o FI foram maiores quando o intervalo aumentou de 30 para 60 s (i.e., da Fase 1 para a Fase 2); e (d) as pausas diminuíram quando uma contingência de $\mathrm{LH}$ foi adicionada ao componente de FI do programa múltiplo. Os autores argumentaram que a sensibilidade do comportamento dos participantes às contingências programadas foi uma função tanto do tipo de reforçador utilizado (que, segundo os autores, permitiu um aumento substancial no salário líquido) quanto pela intercomunicação originada fora da sessão experimental. De qualquer modo, as instruções que os participantes passaram uns para os outros foram produtos das contingências de reforço da sessão experimental e, mesmo recebendo instruções, os resultados indicaram que o comportamento dos participantes fez contato com as contingências da sessão antes de atingir o padrão final. Segundo os autores, "o desempenho final, então, pode ser aceito como conseqüência dos procedimentos experimentais" (Stoddard e cols., 1988, p. 42).

Uma variação de procedimento que pode ser relevante diz respeito à resposta de consumação [consummatory response]. Por exemplo, Matthews e cols. (1977) realizaram um estudo (Experimento II) com 10 universitários expostos a um programa de reforço em FI. Após 25 reforços em CRF, seis participantes tiveram uma curta exposição (50 reforços) em VR antes da contingência ser alterada para um FI 60 s e quatro estudantes foram expostos ao FI, cujo intervalo aumentou progressivamente até atingir um FI 50 s. Diferentemente da maioria dos estudos sobre programas de reforço com humanos, os autores modelaram a resposta de pressão ao botão em vez de estabelecê-la por instrução ${ }^{2}$ e criaram uma resposta de consumação: a cada ponto liberado os participantes tinham de parar de responder no botão de resposta (o operandum) e deviam pressionar outro botão (o botão de resposta de consumação) para que o ponto fosse creditado no contador. Os participantes recebiam U\$ 0,01 para cada ponto ganho na sessão. Este procedimento está mais próximo daqueles utilizados com não-humanos do que os estudos comumente realizados com humanos nos quais a resposta operante é estabelecida via 
instrução e nenhuma resposta de consumação, que interrompa o comportamento em andamento, é exigida. Os resultados indicaram que quando uma contingência de FI foi posta em vigor os participantes tenderam a exibir um padrão de respostas em taxa baixa e alguns padrões de scallop foram observados. Ao final da sessão (90 minutos) o desempenho dos participantes era de uma resposta por intervalo. Esse padrão responder, embora não seja típico do responder de não-humanos sob FI sugere que o desempenho era sensível ao parâmetro temporal do programa de reforço $^{3}$. Os autores argumentaram que, possivelmente, diferenças no procedimento entre os estudos com humanos e não-humanos são responsáveis pelas discrepâncias nos resultados dos estudos sobre programas de reforço.

Em outro experimento de Matthews e cols. (1977, Experimento I) os resultados sugerem que a resposta de consumação e a história de aquisição da resposta operante (i.e., se por instrução, modelagem ou modelação) são variáveis importantes para produzir desempenhos em programas de reforço que repliquem resultados obtidos com não-humanos. Quando sujeitos não-humanos são expostos a programas de VR ou VI e o intervalo entre reforçadores é igualado nos dois programas, uma maior taxa de respostas é observada para os sujeitos expostos ao VR (cf. Catania, 1998). Matthews e cols. expuseram 16 duplas de estudantes universitários a um programa de reforço em VR e em yoked VI. A resposta de pressão ao botão foi modelada ou demonstrada (modelação) e a obtenção dos pontos exigia ou não uma resposta de consumação. As taxas de respostas no VR tenderam a ser mais altas que no yoked VI - um resultado semelhante aos obtidos com organismos não-humanos - quando o responder foi estabelecido por modelagem e uma resposta de consumação era exigida. Quando a resposta de consumação estava ausente, apenas duas de cinco duplas de participantes apresentaram taxas de respostas em VR mais altas do que em VI.

Buskist, Miller e Bennett (1980) submeteram sete estudantes a um programa de reforço em FI e FT utilizando alimento como conseqüência - que também envolvia uma resposta de consumação. A tarefa experimental consistia em abrir uma pequena porta do aparato e verificar se havia um pedaço de fruta seca disponível. Abrir a porta do aparato era a resposta operante estudada, os pedaços de fruta a conseqüência programada e comer as frutas a resposta de consumação. Diferentemente do estudo de Matthews e cols. (1977), a resposta foi estabelecida por instrução e não modelada. Dois participantes exibiram padrão de respostas em taxa alta e constante, três exibiram padrões de taxa baixa e constante e dois participantes exibiram um padrão de responder em scallops. Esse resultado sugere que a resposta de consumação pode ser uma condição importante para facilitar o desenvolvimento de um padrão de responder em humanos que se assemelhe àqueles obtidos com organismos não-humanos respondendo sob FI, mas que esta não é a única condição importante uma vez que um padrão de responder em taxa alta ainda foi mantido para dois dos sete participantes. Portanto, o papel da resposta de consumação requer futuras investigações.

Raia, Shillingford, Miller e Baier (2000) realizaram um estudo (Experimento I) no qual examinaram o desempenho de estudantes sob um programa de reforço em VR e yoked VI em função da combinação de diversas variáveis: tipo de instruções (rica, mínima ou nenhuma $)^{4}$, forma de aquisição do responder (com ou sem modelagem) e resposta de consumação (com ou sem ela). Portanto, 12 grupos foram gerados. Por exemplo, um grupo de participantes foi submetido (aos pares) à condição VR yoked VI com instrução rica, sem modelagem e com resposta de consumação; outro grupo recebeu instrução mínima, com modelagem e com resposta de consumação; outro grupo não recebeu instrução, a resposta operante foi modelada e não era exigida resposta de consumação e assim por diante. Entre outras coisas, os resultados sugeriram que taxas altas em VR e baixas no yoked VI foram obtidas principalmente para os participantes do grupo em que uma resposta de consumação foi exigida, as instruções eram mínimas e a resposta foi adquirida por modelagem - resultados que corroboram aqueles obtidos por Matthews e cols. (1977, Experimento I); inversamente, taxas de respostas quase idênticas entre VR e o yoked VI foram obtidas com os participantes nos quais não havia resposta de consumação e a resposta era estabelecida totalmente por instrução (i.e., sem modelagem e com instruções ricas). Os resultados também sugeriram que o tipo das instruções teve efeito diferencial sobre o responder em VI apenas quando uma resposta de consumação foi exigida. Em outras palavras, o efeito do tipo de instrução (se mais completa, menos completa ou ausente) dependeu de existir ou não uma resposta de consumação. Na condição sem resposta de consumação os três tipos de instrução não produziram taxas de respostas diferenciadas em VI.

Uma vez que (a) a discrepância nos resultados de humanos e não-humanos, bem como a variabilidade 
nos resultados entre humanos tem implicações tanto do ponto de vista teórico-conceitual quanto prático; (b) os efeitos da resposta de consumação sobre o desempenho de humanos em programas de reforço parece ter sido ainda pouco investigada diretamente e os resultados existentes são, aparentemente, ainda controversos e (c) a interação entre o tipo de instrução fornecida e a presença ou não de uma resposta de consumação precisam ser mais bem estudadas, os dois experimentos do presente trabalho investigaram, de maneira geral, o efeito da exigência ou não de uma resposta de consumação sobre o desempenho de humanos sob FI quando as instruções eram mais detalhadas e quando elas eram mínimas.

Assim, o Experimento I, investigou o efeito da resposta de consumação sobre o desempenho de humanos em FI. Entretanto, o grupo de participantes que foi submetido ao FI sem a exigência de uma resposta de consumação recebeu uma instrução mínima enquanto que o grupo de participantes que foi submetido ao FI com a exigência da resposta de consumação recebeu uma instrução que fazia referência a esta resposta (i.e.,os participantes desse grupo tiveram uma instrução “adicional”). Portanto, o resultado do Experimento I, não separou os efeitos da resposta de consumação dos efeitos do tipo de instrução fornecida. O Experimento II foi delineado para isolar o efeito do tipo de instrução daquele da resposta de consumação. No Experimento II para alguns participantes foi exigida uma resposta de consumação e para outros não, mas todos os participantes receberam as mesmas instruções mínimas.
Experimento I: Efeito da exigência de uma resposta de consumação quando uma instrução "adicional” acerca da resposta de consumação foi fornecida para os participantes de um dos grupos.

\section{MÉTODO}

\section{Participantes}

Seis universitários, exceto do curso de Psicologia, de ambos os sexos.

\section{Equipamentos e Instrumento}

Equipamentos: Foram utilizados dois computadores do tipo PC. Um deles com processador 486 e 16 MB de memória RAM e outro com processador Pentium 100 MHZ e 32 MB de memória RAM. Ambos possuíam monitores de 14 polegadas em cores, mouse e teclado padrão. Foram utilizados também dois fones de ouvido da marca Groove, modelo HD-3030 e dois gravadores (um Panasonic Slim Line, RQ-2103 e um Sony TCM 353V).

Instrumento: Foi utilizado o software ProgRef v3.1 (Costa \& Banaco, 2002; 2003). O software foi programado em linguagem Visual Basic ${ }^{\circledR} 6.0$ e é executável em ambiente Windows ${ }^{\circledR}$ (Windows 95, 98 ou 98se). Os participantes deviam clicar com o cursor do mouse sobre um retângulo (botão de resposta) que aparecia na parte central inferior do monitor. Os pontos apareciam em uma janela (visor de pontuação) acima do botão de resposta e eram apresentados de acordo com o programa de reforço selecionado pelo experimentador. A Figura 1 exibe um layout das telas do computador com a qual os participantes interagiram.

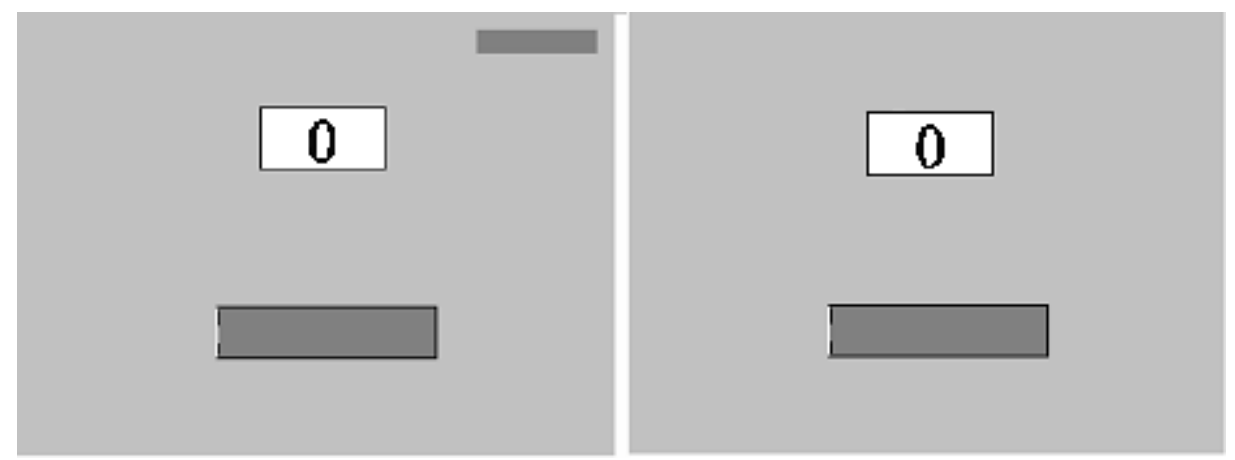

Figura 1. Dois tipos possíveis de telas com as quais os participantes interagiam. A tela da esquerda contém o botão de respostas (retângulo no centro inferior da tela), o visor de pontuação (no centro superior da tela) e o botão de resposta de consumação (no canto superior direito da tela). A tela da direita exibe um layout semelhante, porém sem o botão da resposta de consumação. 


\section{Local}

O experimento foi conduzido no Laboratório de Análise Experimental do Comportamento Humano (LAECH) do Departamento de Psicologia Geral e Análise do Comportamento da UEL. O laboratório mede aproximadamente $24 \mathrm{~m}^{2}$ e possui quatro cubículos com, aproximadamente $3 \mathrm{~m}^{2}$ cada. Os computadores estavam nos dois primeiros cubículos.

\section{Procedimento}

Antes do início da primeira sessão, os participantes assinaram um Termo de Consentimento Livre e Esclarecido que trazia informações sobre as condições para participar do estudo ${ }^{5}$. Foi solicitado aos participantes que deixassem todo material, inclusive relógio, fora do cubículo no qual o computador se encontrava. Foi também solicitado que desligassem o telefone celular caso portassem algum.

Cada participante foi submetido a três sessões experimentais, com 20 minutos de duração cada, sob um programa de reforço em FI $30 \mathrm{~s}$. Sob este programa de reforço o participante ganhava um ponto (ou aparecia um smile) para cada resposta emitida 30 segundos após a liberação do último ponto (ou aparecimento do smile). Ao final de cada sessão o participante recebia R\$ 0,05 para cada ponto obtido. Os participantes foram distribuídos em dois grupos:

Grupo CR-I - Com resposta de consumação $(n=3)$ : Para os participantes desse grupo era exigida uma “resposta de consumação” para cada ponto ganho. Os participantes desse grupo receberam a seguinte instrução por escrito:

Obrigado por sua colaboração! Você deverá clicar no botão esquerdo do mouse com o cursor sobre um retângulo que aparecerá na parte central inferior do monitor. Clicando sobre o botão de maneiras específicas você ganhará pontos. Os pontos aparecerão em uma janela na parte central superior do monitor. Você deve tentar ganhar o maior número de pontos que você conseguir. Os pontos aparecerão na janela que se localiza na parte superior da tela na posição central.

[Entretanto, quando você ganhar algum ponto aparecerá no canto superior direito do monitor um smile. Você deverá, então, clicar com a seta do mouse sobre o botão que se localiza no canto superior direito da tela. Ao fazer isso o smile desaparecerá e o ponto será creditado no contador. Quando um smile aparecer no monitor não aparecerá outro até que você clique no botão menor no canto supe- rior direito. Depois que o smile desaparece - e o ponto é creditado - você pode voltar a clicar sobre o botão maior na parte inferior do monitor para ganhar mais pontos.]

Caso você não tenha entendido as instruções, faça uma nova leitura. Restando dúvidas, informe ao experimentador. Boa sorte!

Quando o participante desse grupo ganhava algum ponto, aparecia no canto superior direito do monitor um ícone identificado comumente como um "smile". Nesse momento, um novo intervalo do FI era iniciado. O participante, então, deveria clicar com a seta do mouse sobre o botão da resposta de consumação. Ao fazer isso o smile desaparecia e o ponto era creditado no contador. Enquanto um smile estivesse presente no monitor não aparecia outro até que o participante pressionasse o botão da resposta de consumação. Depois que o smile desaparecia - e o ponto era creditado - o participante, caso voltasse a pressionar o botão de resposta, poderia receber outro smile. Portanto, o aparecimento do smile "marcava" o final de um intervalo do FI e início do intervalo subseqüente, mas seu desaparecimento não tinha qualquer efeito sobre os intervalos de tempo computados pelo software.

Grupo SR-I - Sem resposta de consumação $(n=3)$ : Para os participantes desse grupo não era exigida uma "resposta de consumação" para que os pontos fossem creditados no visor de pontuação. Os participantes desse grupo receberam, por escrito, uma instrução semelhante a do grupo anterior. A diferença é que a instrução referente à resposta de consumação foi omitida para estes participantes (trecho entre colchetes na instrução acima). Para os participantes desse grupo o botão de resposta de consumação não era visível. Quando a contingência de reforço era cumprida, um ponto era imediatamente creditado no contador e o cronômetro que registrava o intervalo da contingência de FI era reiniciado.

Durante todas as sessões os participantes utilizaram um fone de ouvido no qual soava um "ruído branco” (som de um rádio fora de estação) para efeitos de isolamento acústico.

\section{RESULTADOS}

A Figura 2 apresenta a taxa geral de resposta dos participantes do Grupo CR-I (Com Resposta de Consumação - painel esquerdo) e dos participantes do Grupo SR-I (Sem Resposta de Consumação - painel 
direito) nas três sessões de FI 30 s. Cada valor da taxa geral de resposta foi obtido dividindo-se o número de resposta na sessão pela duração total da sessão.
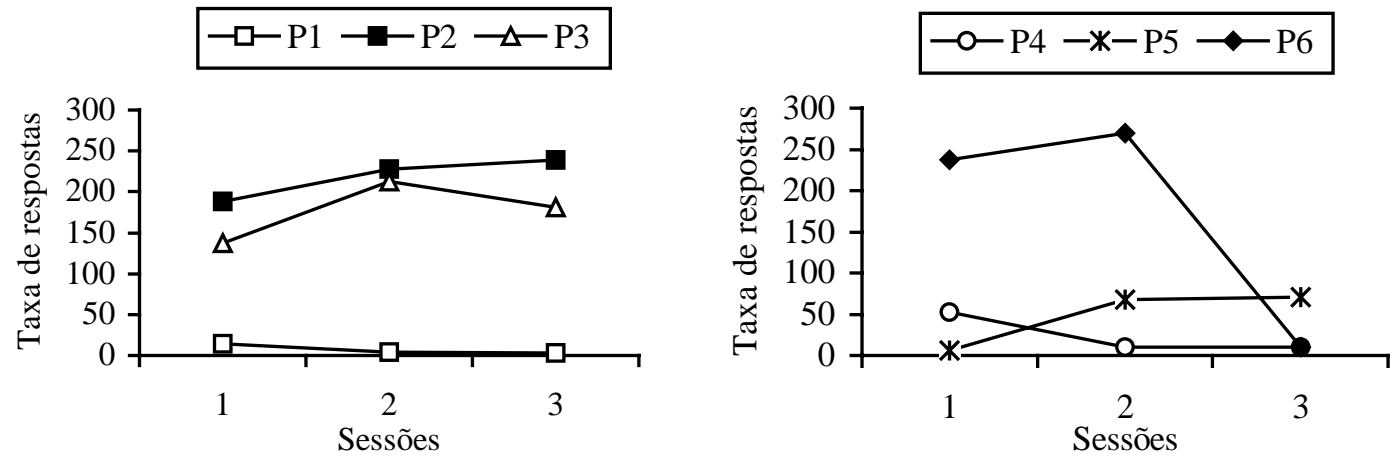

Figura 2. Taxa geral de resposta dos participantes P1, P2 e P3 do Grupo CR-I (painel esquerdo) e dos participantes P4, P5 e P6 do Grupo SR-I (painel direito) nas três sessões de FI $30 \mathrm{~s}$.

Observa-se na Figura 2 que dois dos três participantes do Grupo CR-I apresentaram taxa relativamente alta de resposta ao longo das três sessões experimentais. Houve maior variabilidade na taxa de resposta entre participantes do Grupo SR-I. Esses participantes também apresentaram maior variabilidade na taxa de resposta entre sessões. A taxa de resposta variou, aparentemente, sem uma ordem sistemática. A taxa de resposta de $\mathrm{P} 4$ diminuiu da primeira para a última sessão; a de P5 aumentou da primeira para a última sessão e a de P6 aumentou ligeiramente da primeira para a segunda sessão e diminuiu acentuadamente da segunda para a terceira sessão.

A Figura 3 exibe o registro cumulativo da terceira sessão de exposição ao FI 30 s dos participantes P1, P2 e P3 (Grupo CR-I - Com resposta de Consumação) e dos participantes P4, P5 e P6 (Grupo SR-I - Sem Resposta de Consumação).

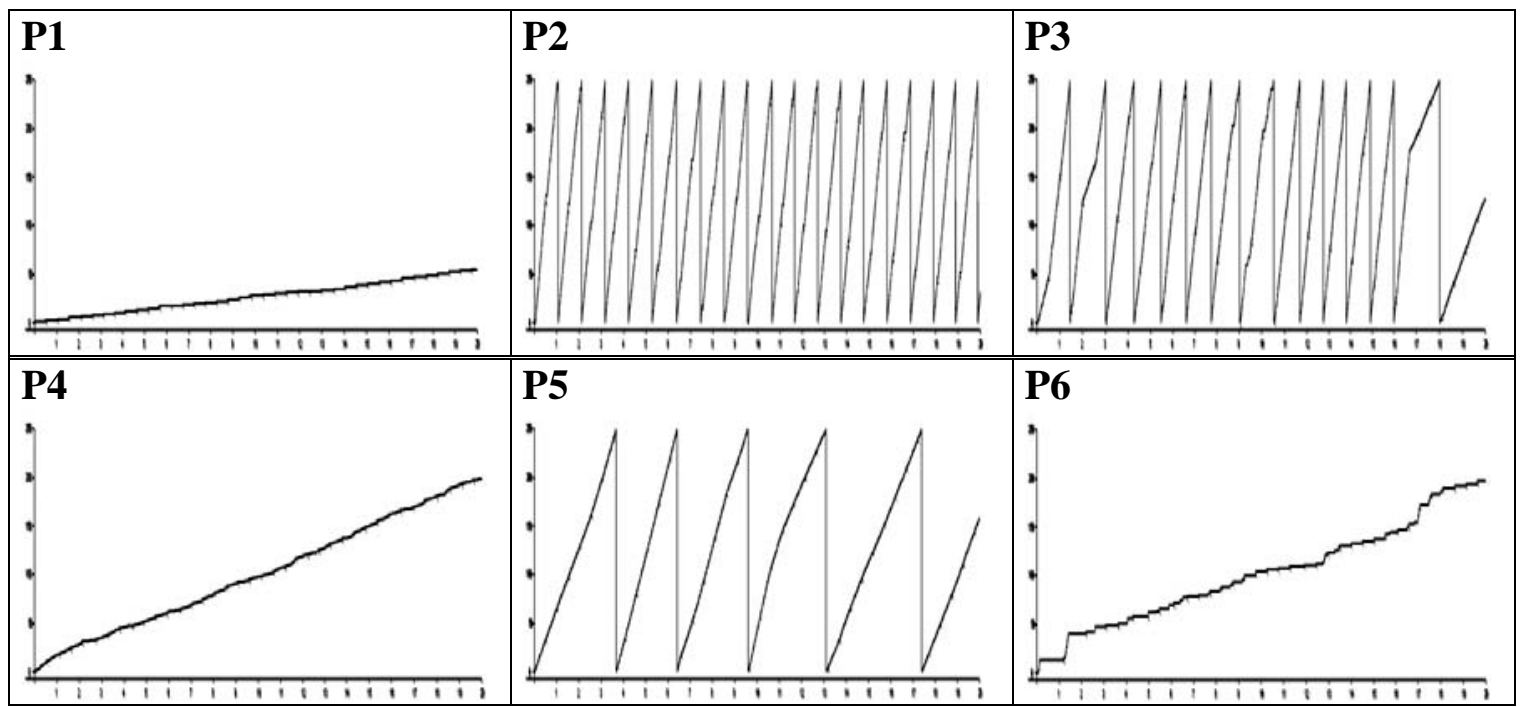

Figura 3. Registro cumulativo de pressão ao botão da terceira sessão de exposição ao FI 30 s dos participantes P1, P2 e P3 (Grupo CR-I porção superior da figura) e dos participantes P4, P5 e P6 (Grupo SR-I - porção inferior da figura). Os registros cumulativos voltam a zero após 250 respostas e as marcas diagonais nos registros indicam a ocorrência de reforço. Todas as sessões tiveram duração de 20 minutos. 
Verifica-se que os participantes do Grupo CR-I exibiram ou um padrão de resposta em taxa baixa e constante entre reforços sucessivos (P1) ou em taxa alta e constante entre reforços sucessivos (P2 e P3), isto é, sem pausa pós-reforço. O padrão da distribuição das respostas entre reforços variou mais entre os participantes do Grupo SR-I. O participante P5 exibiu um padrão de taxas relativamente mais altas do que P4 e P6.

A Figura 4 exibe uma ampliação do registro cumulativo de parte da sessão de P6 (do $13^{\circ}$ ao $19^{\circ} \mathrm{mi}-$ nuto da Sessão 3).

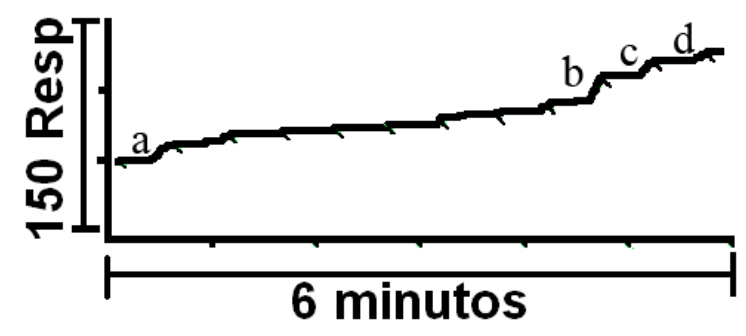

Figura 4. Registro cumulativo do $13^{\circ}$ ao $19^{\circ}$ minutos da terceira sessão de P6.

Verifica-se, através da análise visual do registro cumulativo, que o participante P6 do Grupo SR-I apresentou alguns poucos padrões do tipo break-andrun (letras $a, b, c$ e $d$ na Figura 4).

\section{DISCUSSÃO}

Os resultados do presente experimento - diferentemente daqueles de Matthews e cols. (1977) - não indicaram que a presença de uma resposta de consumação tenha aumentado a probabilidade de um desempenho sob controle do parâmetro temporal da contingência de FI. A principal diferença entre os dois grupos do Experimento I diz respeito à variabilidade no desempenho. Aparentemente, o desempenho dos participantes do Grupo SR-I apresentou maior variabilidade intra e entre participantes do que o desempenho dos participantes do Grupo CR-I (ver Figura 2). Entretanto, mesmo essa diferença não foi marcante, dada a variabilidade na taxa de respostas que se observa entre P1 (baixas taxas) e P2 e P3 (altas taxas) no grupo com resposta de consumação.

Resultados de Raia e cols. (2000) sugerem que o efeito de uma resposta de consumação pode depender da interação com outras variáveis, tais como o tipo de instrução utilizada. Naquele estudo, Raia e cols., observaram que um desempenho sob controle do programa de reforço em vigor era mais aparente para os participantes em que uma resposta de consumação foi exigida, as instruções eram mínimas e a resposta foi adquirida por modelagem. Pode-se especular que, no presente estudo, uma instrução menos detalhada tal- vez pudesse revelar melhor um possível efeito da resposta de consumação. Um problema metodológico do Experimento I foi que, ao receberem uma instrução acerca da resposta de consumação, os participantes do Grupo CR-I receberam uma instrução diferente (e, em certo sentido, mais completa) do que aquela dos participantes do Grupo SR-I. Uma maneira de contornar essa questão - e separar o possível efeito da exigência ou não de uma resposta de consumação de possíveis efeitos de diferentes instruções - seria fornecer uma mesma instrução (mínima) para os participantes de ambos os grupos.

Além das instruções pode-se considerar o tempo de exposição às contingências de reforço. No presente estudo os participantes foram expostos ao FI $30 \mathrm{~s}$ por três sessões de 20 minutos cada (60 minutos no total). Perone e cols. (1988) ressaltaram que os padrões de resposta em FI podem depender, entre outras coisas, do tempo de exposição ao esquema. Com nãohumanos, quanto maior o tempo de exposição ao FI, mais claramente aparecem os padrões "típicos" de desempenho desse programa e reforço. Os autores argumentaram que as variações no desempenho de humanos sob FI podem ser um reflexo de uma curta história de exposição a esta contingência de reforço.

Observou-se, no presente estudo que o participante P3 (Figura 3) reduziu a taxa de resposta nos minutos finais da terceira sessão de exposição ao FI (embora a taxa de resposta continuasse constante nos intervalos entre reforços). É possível especular que, se o tempo de exposição ao FI fosse estendido, a taxa de resposta poderia cair ainda mais. 
Um efeito interessante do uso da resposta de consumação pôde ser observado (de forma assistemática) durante a coleta de dados. Os participantes do Grupo SR-I não mantinham seus olhos voltados para a tela do computador todo o tempo e em algumas ocasiões deitavam a cabeça na mesa e continuavam a pressionar o botão do mouse. Resultados semelhantes foram relatados por Matthews e cols. (1977).

\section{Experimento II: Efeito da exigência de uma res- posta de consumação quando foi utilizada uma instrução mínima.}

O Experimento II procurou investigar algumas questões levantadas a partir dos resultados do Experimento I: o efeito da resposta de consumação seria diferente se fosse fornecida uma única instrução (mínima) para ambos os grupos? Aumentar o tempo de exposição ao FI forneceria resultados mais claros do que aqueles obtidos no Experimento I? No Experimento II o tempo total de exposição ao FI foi aumentado para 90 minutos e uma mesma instrução foi fornecida para os participantes para quem era exigida uma resposta de consumação e para os participantes para quem tal resposta não era exigida.

\section{MÉTODO}

\section{Participantes}

Oito universitários, exceto do curso de Psicologia, de ambos os sexos.

\section{Equipamentos e Instrumento}

Os equipamentos e instrumento foram os mesmos do Experimento I.

\section{Local}

O mesmo do Experimento I.

\section{Procedimento}

Antes do início da primeira sessão, os participantes assinaram um Termo de Consentimento Livre e Esclarecido que trazia informações sobre as condições para participar do estudo ${ }^{6}$. Foi solicitado aos participantes que deixassem todo material, inclusive relógio, fora do cubículo no qual o computador se encontrava. Foi também solicitado que desligassem o telefone celular caso portassem algum.

Cada participante foi submetido a três sessões experimentais, com duração de 30 minutos cada, sob um programa de reforço em FI 30 s. Ao final de cada sessão o participante recebia $\mathrm{R} \$ 0,05$ para cada ponto obtido. Os participantes foram distribuídos em dois grupos:

Grupo CR-II - Com resposta de consumação $(n=4)$ : Para os participantes desse grupo foi exigida uma "resposta de consumação" para cada ponto ganho. Os participantes receberam a seguinte instrução por escrito:

Obrigado por sua colaboração! Você deverá manipular o mouse de alguma maneira para tentar ganhar o maior número de pontos que você conseguir. Os pontos serão acumulados em uma janela na parte central superior do monitor. Caso você não tenha entendido as instruções, faça uma nova leitura. Boa sorte!

Essa instrução foi construída a partir das instruções fornecidas ao Grupo SR-I do Experimento I. Segue, abaixo, a transcrição do trecho inicial da instrução fornecida aos participantes do Grupo SR-I com marcas que indicam as modificações realizadas.

Obrigado por sua colaboração! Você deverá clicar no botão esquerdo do mouse com o cursor sobre um retângulo que aparecerá na parte central inferior do monitor. Clicando sobre o botão de maneiras específicas você ganhará pontos. Os pontos aparecerão em uma janela na parte central superior do monitor. Você deve tentar ganhar o maior número de pontos que você conseguir. Os pontos aparecerão na janela que se localiza na parte superior da tela na posição central.

As partes em negrito foram mantidas inalteradas; as partes grifadas foram mantidas com alteração na redação e as demais partes foram suprimidas com a inserção de outras. A mudança mais importante entre a instrução fornecida ao Grupo SR-I do Experimento I e a instrução do Experimento II diz respeito à descrição (geral) da topografia da resposta para se obter os pontos que aparece explicitamente no Experimento I e de modo menos direto no Experimento II (comparar trechos com sublinhado duplo, em ambas as instruções).

Grupo SR-II - Sem resposta de consumação $(n=4)$ : Para os participantes desse grupo não foi exigida uma "resposta de consumação". Os participantes receberam a mesma instrução do Grupo CR-II.

Durante todas as sessões os participantes utilizaram um fone de ouvido no qual soava um "ruído branco” (som de um rádio fora de estação) para efeitos de isolamento acústico. 


\section{RESULTADOS}

A Figura 5 apresenta a taxa geral de resposta dos participantes do Grupo CR-II (Com Resposta de Consumação - painel esquerdo) e dos participantes do

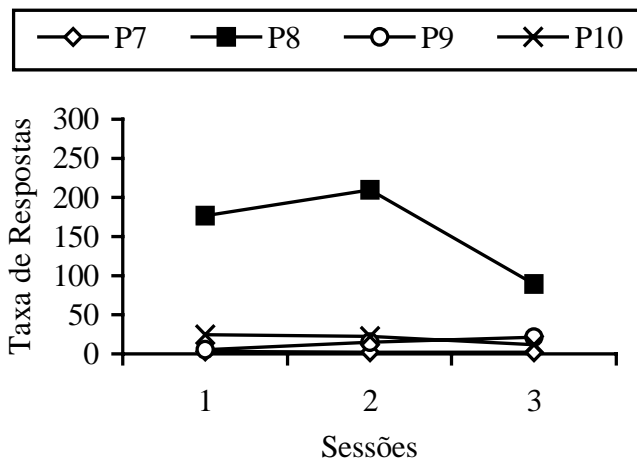

Grupo SR-II (Sem Resposta de Consumação - painel direito) nas três sessões de FI 30 s. Cada valor da taxa geral de resposta foi obtido dividindo-se o número de resposta na sessão pela duração total da sessão.

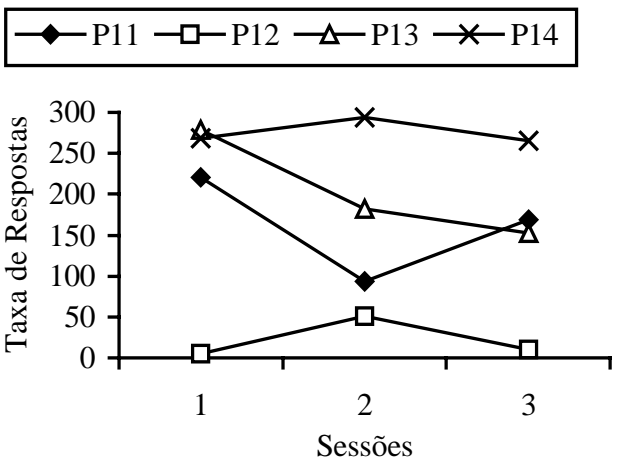

Figura 5. Taxa geral de resposta dos participantes P7, P8, P9 e P10 do Grupo CR-II (painel esquerdo) e dos participantes P11, P12, P13 e P14 do Grupo SR-II (painel direito) nas três sessões de FI 30 s.

Apenas um participante do Grupo CR-II (P8), para quem era exigida a resposta de consumação, apresentou um desempenho em taxa alta de resposta durante a exposição ao FI 30 s. Mesmo assim, o desempenho na terceira sessão foi de taxa abaixo das sessões anteriores. Os demais participantes apresentaram um desempenho de baixa taxa sob o FI. Três dos quatro participantes do Grupo SR-II (P11, P13 e P14), que responderam sem a exigência da resposta de consumação, apresentaram alta taxa de resposta. Apenas o participante P12 desse grupo apresentou um desempenho em taxa baixa.

A Figura 6 exibe o registro cumulativo da terceira sessão de exposição ao FI 30 s dos participantes P7, P8, P9 e P10 (Grupo CR-II - Com resposta de Consumação) e dos participantes P11, P12, P13 e P14 (Grupo SR-II - Sem Resposta de Consumação).

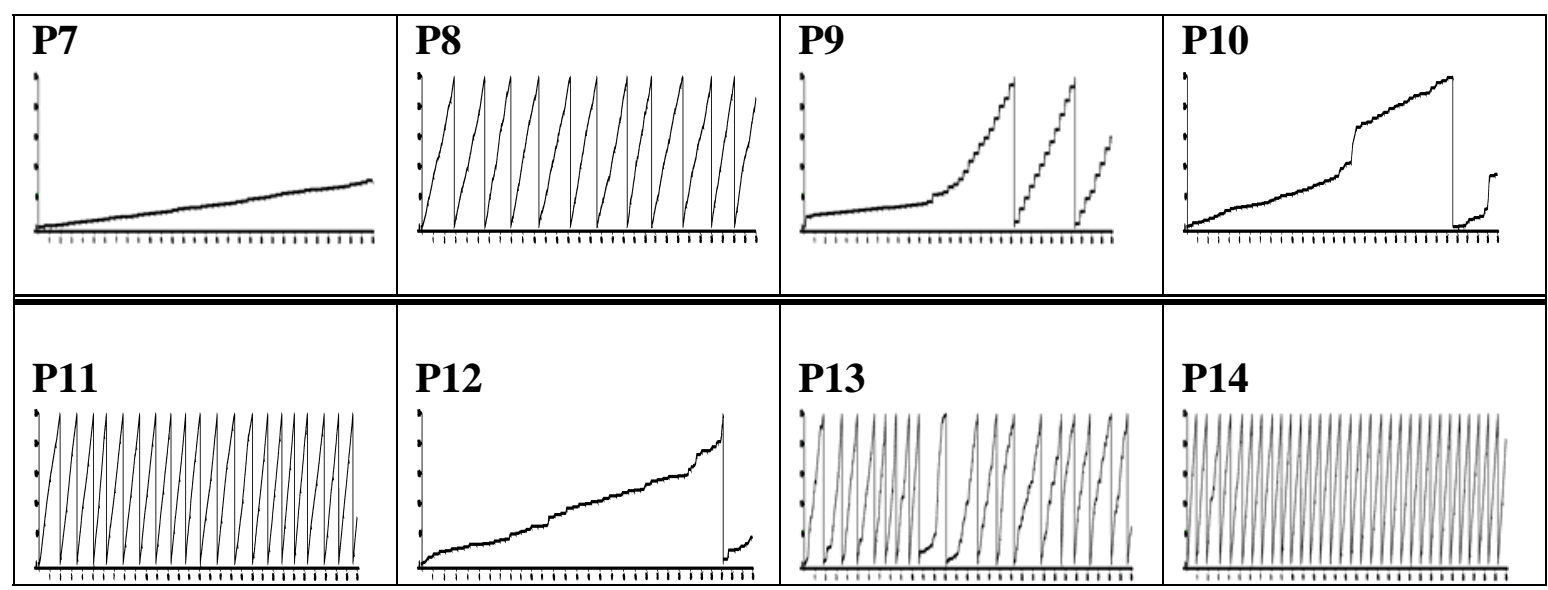

Figura 6. Registro cumulativo de pressão ao botão da terceira sessão de exposição ao Fl 30 s dos participantes P7, P8, P9 e P10 (Grupo CR-II - porção superior da figura) e dos participantes P11, P12, P13 e P14 (Grupo SR-II - porção inferior da figura). Os registros cumulativos voltam a zero após 250 respostas. Todas as sessões tiveram duração de 30 minutos.

Pode-se observar na Figura 6 que o desempenho de P7, P9 e P10 pareciam sob controle do parâmetro temporal da contingência de FI. O participante P7 exibiu um desempenho de taxa baixa, com geralmente 
uma resposta por intervalo entre reforços. O participante P9 exibiu um padrão de baixa taxa (parecido com o P7) no primeiro terço da sessão; depois passou a exibir um padrão de break-and-run (pausa pósreforço seguida por uma taxa alta de resposta que se mantinha até o próximo reforço). O P10 exibiu um padrão de respostas em taxa baixa (em geral entre 2 a 5 respostas por intervalo entre reforços), intercalado com curtos períodos de responder em taxa alta (como, por exemplo, por volta da metade e quase no final da sessão). O participante P8 foi o único desse grupo que apresentou um padrão de desempenho de taxa alta de respostas que se manteve por toda a sessão. Todavia, a taxa de respostas de P8 na terceira sessão de exposição ao FI foi mais baixa que nas sessões anteriores como pôde ser visto na Figura 5.

Três dos quatro participantes do grupo submetido ao FI sem a exigência de uma resposta de consumação (porção inferior da Figura 6) apresentaram um padrão de responder em taxa alta (P11, P13 e P14). O participante P13 exibiu, ocasionalmente, um desempenho em taxa baixa, mas que não se manteve ao longo da sessão. Apenas o desempenho do participante P12 pareceu sob controle do parâmetro temporal da contingência de FI.

\section{DISCUSSÃO}

Os resultados do Experimento II do presente estudo parecem indicar que a exigência de uma resposta de consumação em um programa de reforço em FI com humanos pode aumentar a probabilidade de um desempenho em taxas baixas, aparentemente sob controle do parâmetro temporal da contingência de FI. Nesse sentido, os resultados do Experimento II corroboram aqueles obtidos por Matthews e cols. (1977). Entretanto, o desempenho em altas taxas de P8 (Grupo CR-II) e o desempenho em baixas taxas de P12 (Grupo SR-II) indicam que o emprego de uma instrução mínima e resposta de consumação, não garantem um desempenho em FI que seja sensível ao parâmetro temporal dessa contingência.

\section{DISCUSSÃO GERAL}

Tomados em conjunto, os resultados dos Experimentos I e II do presente estudo parecem indicar que o efeito da resposta de consumação sobre a taxa de respostas pode depender da interação com outras variáveis, tais como, o tipo de instrução. Quando os participantes, para quem foi exigida a resposta de consumação, receberam uma instrução "adicional” acerca da resposta de consumação e uma descrição mais explícita da topografia da resposta para se obter os pontos (i.e., "clicar no botão esquerdo do mouse com o cursor sobre um retângulo que aparecerá na parte central inferior do monitor" - Experimento I) o desempenho não pareceu mais provável de ficar sob controle do parâmetro temporal da contingência de FI. Quando a instrução "adicional” foi removida (Experimento II) e todos os participantes receberam instruções mínimas acerca da tarefa experimental e uma descrição mais geral da topografia da resposta (i.e., "Você deverá manipular o mouse de alguma maneira”) a resposta de consumação pareceu exercer um efeito diferencial mais claro. Tais resultados parecem apontar na mesma direção daqueles de Raia e cols. (2000). Naquele estudo, o tipo das instruções teve efeito diferencial quando uma resposta de consumação foi exigida. No presente estudo, a presença ou ausência de uma resposta de consumação teve efeito quando um tipo específico de instrução foi utilizado (i.e., instrução mínima). Em comum, os estudos apontam para a importância de se considerar a interação entre diversos aspectos do arranjo experimental (e.g., instruções e exigência de resposta de consumação) nos estudos de humanos respondendo sob FI.

O tempo de duração da sessão não pareceu uma variável importante no presente trabalho. No Experimento I os participantes foram expostos a três sessões de 20 minutos cada uma (i.e., 60 minutos de exposição total ao FI). No Experimento II os participantes foram expostos a três sessões de 30 minutos cada uma (i.e., 90 minutos de exposição total ao FI). Observouse que nos últimos 30 minutos os desempenhos dos participantes dos dois grupos do Experimento II eram diferentes entre si (i.e., no geral, taxas baixas para os que tinham uma resposta de consumação e taxas altas para os que não tinham de emitir uma resposta de consumação). Teriam sido estes resultados visíveis apenas porque o tempo de exposição ao FI no Experimento II foi maior do que o tempo de exposição no Experimento I? Aparentemente não. Era possível observar a diferença entre os grupos do Experimento II já na segunda sessão do FI. Ou seja, mesmo que o tempo de exposição ao FI dos participantes do Experimento II fosse reduzido para 60 minutos, já seria possível observar os efeitos relatados no presente estudo. De qualquer modo uma pergunta ainda permanece: maior tempo de exposição ao FI teria contribuído para a redução das diferenças entre os grupos? A taxa de resposta dos participantes do Grupo SR-II do Experimento II teria diminuído e, eventualmente, 
ficado sob controle do parâmetro temporal do FI se a exposição a esta contingência fosse ainda mais prolongada? Pesquisas que investiguem diretamente esta questão necessitam ser conduzidas para lançar luz sobre o efeito de mais esta variação de procedimento, aumentando assim nosso entendimento do controle do comportamento humano no setting de pesquisas experimentais com programas de reforço.

Costa e cols. (2005) sugeriram que a conseqüência programada para o responder (pontos trocáveis por dinheiro, por exemplo) pode aumentar a probabilidade de um desempenho em taxa alta e constante sob FI. Naquele estudo todos os participantes tinham de emitir uma resposta de consumação e as instruções fornecidas aos participantes foram semelhantes àquelas fornecidas aos participantes do Grupo CR-I do Experimento I do presente estudo, cujos pontos também foram trocados por dinheiro. Ambos os estudos fornecem resultados semelhantes nessas condições. Entretanto, os resultados do presente estudo sugerem que quando instruções mínimas são fornecidas e uma resposta de consumação é exigida, é mais provável que o comportamento dos participantes fique sob controle do FI mesmo quando os pontos são trocáveis por dinheiro.

Os resultados do presente estudo parecem somar ao de outros pesquisadores (e.g., Costa, 2004; Costa e cols., 2005; Matthews e cols., 1977, Raia e cols., 2000; Stoddard e cols., 1988) para indicar que o desempenho de humanos sob FI - que diferem dos padrões relatados de organismos não-humanos - pode ser originado por fatores de procedimento que ainda necessitam de maior atenção e estudos (cf. Perone e cols., 1988).

\section{REFERÊNCIAS}

Bentall, R. P., Lowe, C. F., \& Beasty, A. (1985). The role of verbal behavior in human learning: II. Developmental differences. Journal of the Experimental Analysis of Behavior, 43(2), 165-180.

Buskist, W. F., Bennett, R. H., \& Miller, H. L. (1981). Effects of instructional constraints on human fixed-interval performance. Journal of the Experimental Analysis of Behavior, 35(2), 217225.

Buskist, W. F., Miller, H. L., \& Bennett, R. H. (1980). Fixedinterval performance in humans: Sensitivity to temporal parameters when food is the reinforcer. Psychological Record, 30(1), 111-121.

Catania, A. C. (1998). Learning (4 ${ }^{\mathrm{a}}$ ed.). New Jersey: Prentice Hall.
Costa, C. E. (2004). A natureza do reforçador como uma variável moduladora dos efeitos da história de reforço sobre o comportamento de seres humanos. Tese de doutorado não publicada, Instituto de Psicologia da Universidade de São Paulo.

Costa, C. E., \& Banaco, R. A. (2002). ProgRef v3: Sistema computadorizado para coleta de dados sobre programas de reforço com humanos - Recursos básicos. Revista Brasileira de Terapia Comportamental e Cognitiva, 4(2), 173-192.

Costa, C. E., \& Banaco, R. A. (2003). ProgRef v3: Sistema computadorizado para coleta de dados sobre programas de reforço com humanos - Recursos adicionais. Revista Brasileira de Terapia Comportamental e Cognitiva, 5(2), 219-229.

Costa, C. E., Banaco, R. A., \& Becker, R. M. (2005). Desempenho em FI com humanos: Efeitos do tipo de reforçador. Temas em Psicologia, 13(1), 18-33. Retirado em 20 de outubro de 2007, de http://www.sbponline.org.br/revista2/vol13n1/PDF/ v13n01a04.pdf

Cumming, W. W., \& Schoenfeld, W. N. (1958). Behavior under extended exposure to a high-value fixed interval reinforcement schedule. Journal of the Experimental Analysis of Behavior, 1, 245-263.

Dews, P. B. (1978). Studies on responding under fixed-interval schedules of reinforcement: II. The scalloped pattern of the cumulative record. Journal of the Experimental Analysis of Behavior, 29(1), 67-75.

Ferster, C. B., \& Skinner, B. F. (1957). Schedules of reinforcement. New York: Appleton-Century-Crofts.

Lattal, K. A. (1991). Scheduling positive reinforcers. Em I. H. Iversen \& K. A. Lattal (Orgs.), Experimental analysis of behavior, part 1 (pp. 87-134). New York, NY: Elsevier Science.

Lowe, C. F. (1979). Determinants of human operant behaviour. Em M. D. Zeiler \& P. Harzem (Orgs.), Reinforcement and the organization of behavior (pp. 159-192). New York: John, Wiley \& Sons.

Matthews, B. A., Shimoff, E., Catania, A. C., \& Sagvolden, T. (1977). Uninstrued human responding: Sensitivity to ratio and interval contingencies. Journal of the Experimental Analysis of Behavior, 27(3), 453-467.

Okouchi, H. (2002). Individual differences in human fixedinterval performance. Psychological Record, 52(2), 173-186.

Perone, M., Galizio, M., \& Baron, A. (1988). The relevance of animal-based principles in the laboratory study of human operant conditioning. Em G. Davey \& C. Cullen (Orgs.), Human operant conditioning and behavior modification (pp. 59-85). Chichester, England: John Willey \& Sons.

Raia, C. P., Shillingford, S. W., Miller, H. L., Jr., \& Baier, P. S. (2000). Interaction of procedural factors in human performance on yoked schedules. Journal of the Experimental Analysis of Behavior, 74(3), 265-281.

Stoddard, L. T., Sidman, M., \& Brady, J. V. (1988). Fixed-interval and fixed ratio reinforcement schedules with human subjects. The Analysis of Verbal Behavior, 6, 33-44.

Wanchisen, B. A. (1990). Forgetting the lessons of history. Behavior Analyst, 13(1), 31-37.

Wanchisen, B. A., Tatham, T. A., \& Mooney, S. E. (1989). Variable-ratio conditioning history produces high- and low-rate 
fixed-interval performance in rats. Journal of the Experimental Analysis of Behavior, 52(2), 167-179.

Weiner, H. (1964). Conditioning history and human fixed-interval performance. Journal of the Experimental Analysis of Behavior, 7(5), 383-385.

Weiner, H. (1965). Conditioning history and maladaptative human operant behavior. Psychological Reports, 17(3), 935-942.

Weiner, H. (1969). Controlling human fixed-interval performance. Journal of the Experimental Analysis of Behavior, 12(3), 349373.
Weiner, H. (1983). Some thoughts on discrepant human-animal performances under schedules of reinforcement. Psychological Record, 33(4), 521-532.

\section{Nota:}

${ }^{1}$ Em alguns estudos o padrão break-and-run é considerado como uma variação do scallop (Dews, 1978) e denominado como tal. Embora Ferster e Skinner (1957) relatem intervalos que mostram uma transição abrupta entre a pausa e uma taxa constante de resposta, não há uma denominação específica para este tipo de transição. A denominação break-and-run aparece pela primeira vez em Cumming e Schoenfeld (1958).

${ }^{2}$ Em ambos os casos houve uma instrução mínima, por escrito. Para maiores detalhes ver Matthews e cols. (1977, p. 455).

${ }^{3}$ Weiner (1983) argumentou que não há porque afirmar que um padrão de desempenho em scallop ou break-and-run (como aqueles exibidos por não-humanos) seja considerado mais sensível do que um padrão de pausa pós-reforço seguido por uma única resposta no intervalo. Em uma relação custo-benefício, esse último padrão - muitas vezes observado no desempenho de humanos - é "mais adaptado” do que os outros dois padrões descritos.

${ }^{4}$ A instrução rica descrevia, em linhas gerais, que os participantes deveriam ganhar o maior número de pontos possíveis e que ganhariam pontos pressionando o botão de um joystick para abater uma nave inimiga. As naves inimigas possuíam um campo de força invisível que permaneceria ligado por um certo período de tempo. A instrução mínima não trazia a descrição sobre as naves inimigas, limitandose a informar que o participante deveria ganhar o maior número de pontos possíveis.

${ }^{5}$ O Experimento I foi aprovado pelo Comitê de Ética em Pesquisas com Seres Humanos da UEL. Parecer CEP: 197/04.

${ }^{6}$ O Experimento II foi aprovado pelo Comitê de Ética em Pesquisas com Seres Humanos da UEL. Parecer CEP: 201/05.

\section{Sobre os autores:}

Carlos Eduardo Costa: Doutor em Psicologia Experimental pela Universidade de São Paulo. Professor do Departamento de Psicologia Geral e Análise do Comportamento e do Programa de Mestrado em Análise do Comportamento da Universidade Estadual de Londrina.

Carlos Henrique Patsko: Aluno do curso de graduação em Psicologia da Universidade Estadual de Londrina.

Rodrigo Morande Becker: Aluno do curso de graduação em Psicologia da Universidade Estadual de Londrina.

Endereço para correspondência: Carlos Eduardo Costa: Rua da Lapa, 498 - Apto 101 - Jardim Higienópolis - 86015-050 - Londrina/PR - Endereço eletrônico: caecosta@uel.br. 